\title{
Interaction Between Nitrogen And Hydrocarbons In Magnetized Plasmas
}

\author{
Adam Cwiklinski*, Andrey Markin ${ }^{\dagger}$, Mandy Baudach ${ }^{* *}$ and Werner Bohmeyer ${ }^{\ddagger}$ \\ *Department of Physics, Freie Universität Berlin, Arnimallee 14, 14195 Berlin \\ ${ }^{\dagger}$ Institute of Physical Chemistry and Electrochemistry, Russian Academy of Sciences, Leninsky pr. 31, 119991 \\ Moscow, Russ. Fed. \\ ** Department of Physics, Humboldt Universität zu Berlin, Newtonstr. 15, 12489 Berlin \\ ${ }^{\ddagger}$ Max-Planck-Institut für Physik, EURATOM Association, Wendelsteinstr. 1, 17491 Greifswald
}

\begin{abstract}
The deposition of amorphous hydrocarbon (a-C:H) films and the influence of nitrogen on these films were studied. The experiments were performed in low temperature plasmas with $T_{e}=3 \ldots 6 \mathrm{eV}$ and $n_{e}=1 \cdot 10^{16} \ldots 4 \cdot 10^{17} \mathrm{~m}^{-3}$. The ratio between injected nitrogen and methane was varied as well as the substrate temperature and the neutral gas pressure. Additionally, the deposition distribution as a function of distance was determined. In situ measurements of the a-C:H growth rate show that the carbon redeposition can be suppressed completely by nitrogen injection (scavenger effect).
\end{abstract}

Keywords: scavenger effect, nitrogen, plasma wall interaction, hydrocarbon films, a-C:H

\section{INTRODUCTION}

Since CFC materials will be used in the high heat flux divertor region, tritium enriched amorphous hydrocarbon (a-C:T) films will be formed resulting from plasma wall interaction and the interaction between plasma and eroded divertor material. The in-vessel tritium inventory will be a crucial constraint for long term operation in ITEER. For safety reasons, too high an inventory requires a major cleaning procedure which is to be postponed as long as possible or - ideally - to be avoided completely.

Besides other methods [1], it came up that nitrogen may be a possible candidate for reducing the deposition of a-C:T films due to the scavenger effect [5]. In the subsequent section, the experiments and their results are presented which aimed to investigate film growth behavior remote from a hydrogen plasma as a function of methane and nitrogen injection.

\section{EXPERIMENTAL}

In the linear plasma device PSI-2 [2,4] hydrocarbon layer formation can be studied on large time scales (hours). A schematic is depicted in Fig. 1 on the 1.h.s. In the discharge region the plasma is generated from 100 sccm hydrogen and flows magnetically confined on a neutralizer plate made from tungsten.

Experiments performed in the target chamber utilized the setup depicted in Fig. 1 on the right. A quadrupole mass spectrometer (QMS1) attached to the target chamber allowed determining the concentration of stable neutral species. A second QMS2 was situated in the exhaust of the plasma device. $0.5 \ldots 2 \mathrm{sccm}$ methane and nitrogen were injected through two nozzles, one of which allowing the injection through the plasma in the direction of two silicon wafers mounted on a heatable holder. A manipulator allowed moving the wafers to and away from the plasma edge. A screen attached to the holder aimed to inhibit ionic species to reach the wafers. For in situ film thickness measurements spectral reflectometry was used. The measurements were performed on a silicon wafer pre-coated with an a-C:H film of roughly $100 \mathrm{~nm}$ in thickness.

For simulating conditions similar to those expected in the pump ducts in ITER, experiments were performed with the setup illustrated in Fig. 2 on the left. A so called hot liner made of stainless steel (SS) surrounded the plasma column and was heated to about $200^{\circ} \mathrm{C}$ by the plasma radiation. Thereby, during methane injection no a-C:H was formed on the hot liner walls because erosion due to atomic hydrogen dominated. Controlled conditions with respect to preventing hydrocarbon losses due to cold walls in the injection region could be achieved. Perpendicular to the hot liner followed the pump duct region with a window for in situ spectral light reflectometry. A clean reference $\mathrm{Si}$ wafer 
and a pre-coated wafer were mounted on a heatable holder $180 \mathrm{~mm}$ from the plasma column axis. Seven additional clean wafers were mounted on a rod in the pump duct for determining the remote deposition distribution by ex situ measurements of the formed films.
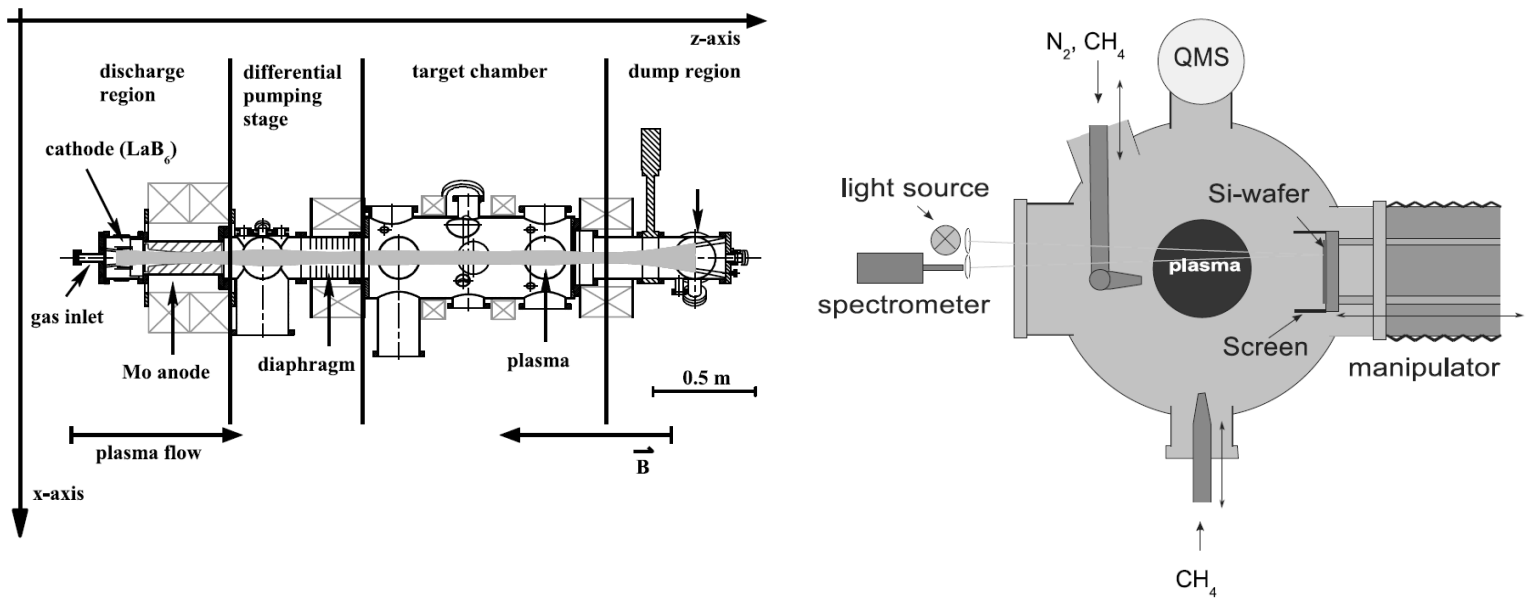

FIGURE 1. Left: Schematic of the linear plasma device PSI-2. Right: Experimental setup in the target chamber.

At the end of the pump duct a turbo molecular pump was attached followed by QMS2 in the exhaust. QMS1 allowed evaluating the amount of species leaving the hot liner into the target chamber through the diaphragm. The plasma conditions for the target chamber experiments were $n_{e}=4 \cdot 10^{17} \mathrm{~m}^{-3}, T_{e}=4.2 \mathrm{eV}$ and $p=0.1 \mathrm{~Pa}$ and for the pump duct experiments the parameters were in the SS liner $n_{e}=1 \cdot 10^{16} \mathrm{~m}^{-3}, T_{e}=5.7 \mathrm{eV}$ and $p=0.1 \mathrm{~Pa}$ and $n_{e}=2 \cdot 10^{17} \mathrm{~m}^{-3}, T_{e}=3.1 \mathrm{eV}$ and $p=1 \mathrm{~Pa}$, respectively. The high pressure conditions were achieved by adding $100 \mathrm{sccm}$ hydrogen through the nozzle to the plasma.
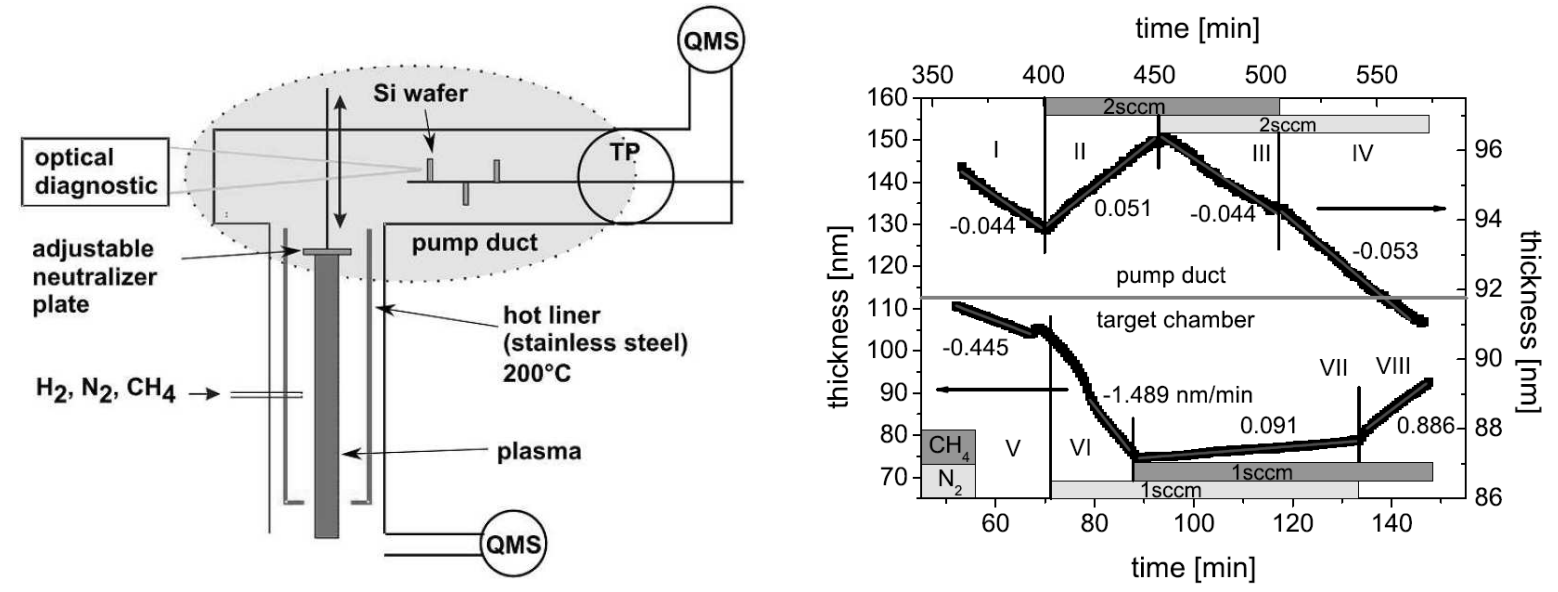

FIGURE 2. Left: Schematic of the pump duct. Right: Growth behavior in the pump duct and in the target chamber at $60^{\circ} \mathrm{C}$ substrate temperature.

\section{RESULTS AND DISCUSSION}

The target chamber experiments aimed to determine how nitrogen influences the film growth at plasma facing surfaces while the experiments performed in the pump duct aimed to suppress possible effects of energetic particle bombardment on the carbon redeposition behavior.

The target chamber and the pump duct experiments showed different behavior with respect to erosion upon nitrogen injection. As depicted in Fig. 2 on the r.h.s., the erosion in the target chamber experiments was enhanced upon nitrogen injection as compared to erosion by atomic hydrogen only (V and VI in the figure). In contrast, in the pump duct 
experiments the erosion rate did not change upon nitrogen injection (I and IV). It is noticed that the data from the target chamber experiments were taken with and without a cylindrical screen aiming to protect the specimen from possible ionic effects. The results did not depend on whether or not the screen was mounted. Possible influence of energetic neutral particle bombardment on erosion should therefore be assessed.

Simultaneous injection of methane and nitrogen led in the target chamber experiment to reduced growth (VII) as compared to the case where methane was injected only (VIII). In the pump duct experiments the film growth (II) was even suppressed completely when nitrogen and methane were injected simultaneously (III). The cause for this behavior have to be volume reactions between nitrogen and hydrocarbon species (cf. I,IV and V,VI), i.e. scavenging, because chemical sputtering [3] seems to be inefficient under the conditions present in the pump duct experiments.
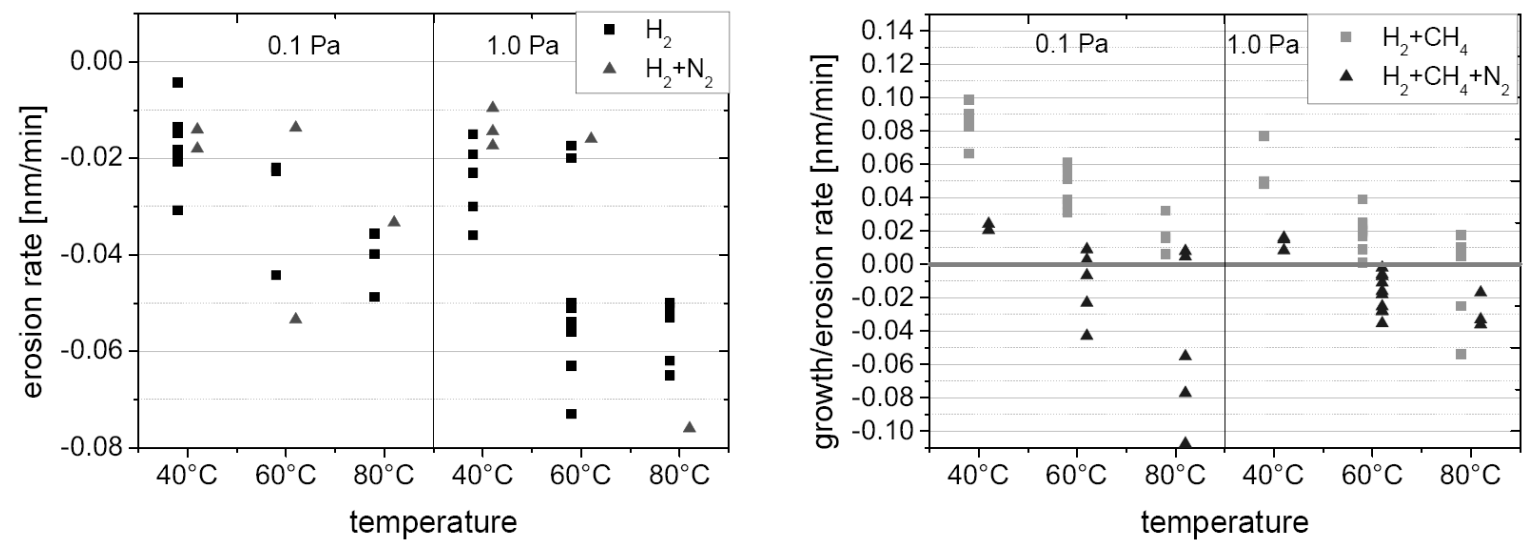

FIGURE 3. Left: Erosion in the pump duct with and without added nitrogen. Right: Growth and erosion rates in the pump duct upon methane injection with and without added nitrogen.

The influence of substrate temperature and neutral gas pressure on film growth and erosion are shown in Fig. 3 for the pump duct experiments. In all cases, the erosion clearly increases with increasing substrate temperature which is in accordance with [6]. High pressure conditions hardly shift down the rates towards more erosion.

The growth rate dependence on the ratio between nitrogen and methane was studied in the pump duct setup with a substrate heated to $40^{\circ} \mathrm{C}$ at low pressure conditions. Net deposition can be switched to net erosion by increasing the nitrogen amount even at low substrate temperatures (Fig. 4, left).

Two experiments were performed for evaluating the effect of nitrogen admission on film distribution along the duct. Seven silicon wafers were mounted onto a long SS holder kept at room temperature. The deposit was analyzed ex situ by a similar optical diagnostics. The resutls are shown in Fig. 4 (right). Similar to the in situ data, the nitrogen admission strongly reduces film growth.

QMS signal evolution with time and the corresponding film growth are exemplified in Fig. 5. The table in Fig. 5 shows that unambiguous assignment of masses to specific species is not simple. Especially if nitrogen and hydrocarbons are present at the same time, reactions between those species result in complicated correlations. Interestingly, when both methane and nitrogen are injected, for instance masses 15 and 16 need much longer to achieve stable signals that it is the case for methane injection only.

\section{CONCLUSION}

Nitrogen admission enhances the erosion at plasma facing surfaces while leaves the erosion unchanged far away from the plasma. Elevating the substrate temperature leads to reduction of the growth rate. At a certain temperature, dependent on the methane flow rate, the deposition changes to erosion which is in accordance with [6]. Nitrogen admission shifts the equilibrium between carbon deposition and $\mathrm{H}$ erosion to lower temperature. This can be regarded as removing/recombining reactive hydrocarbon radicals in reactions with $\mathrm{N}$ species, i.e. scavenging.

As the effect of nitrogen on growth rate is rather strong, application in reactors has to be considered carefully. If nitrogen can be applied such that it does not harm the CFC plates it may very well contribute to reducing the film growth in the pump ducts and thus reduce the amount of co-deposited tritium. 

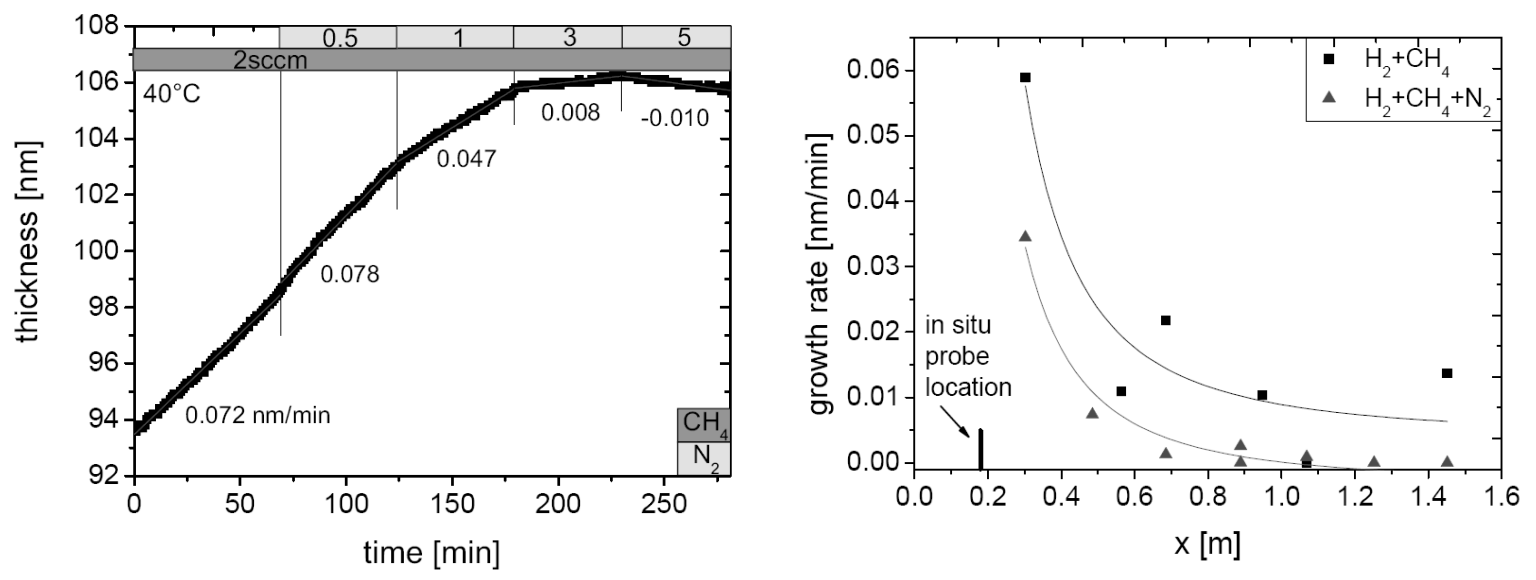

FIGURE 4. Left: Effect of nitrogen content on film growth. Right: Growth rate distribution along the duct ( $\mathrm{x}$ - distance from the plasma axis).

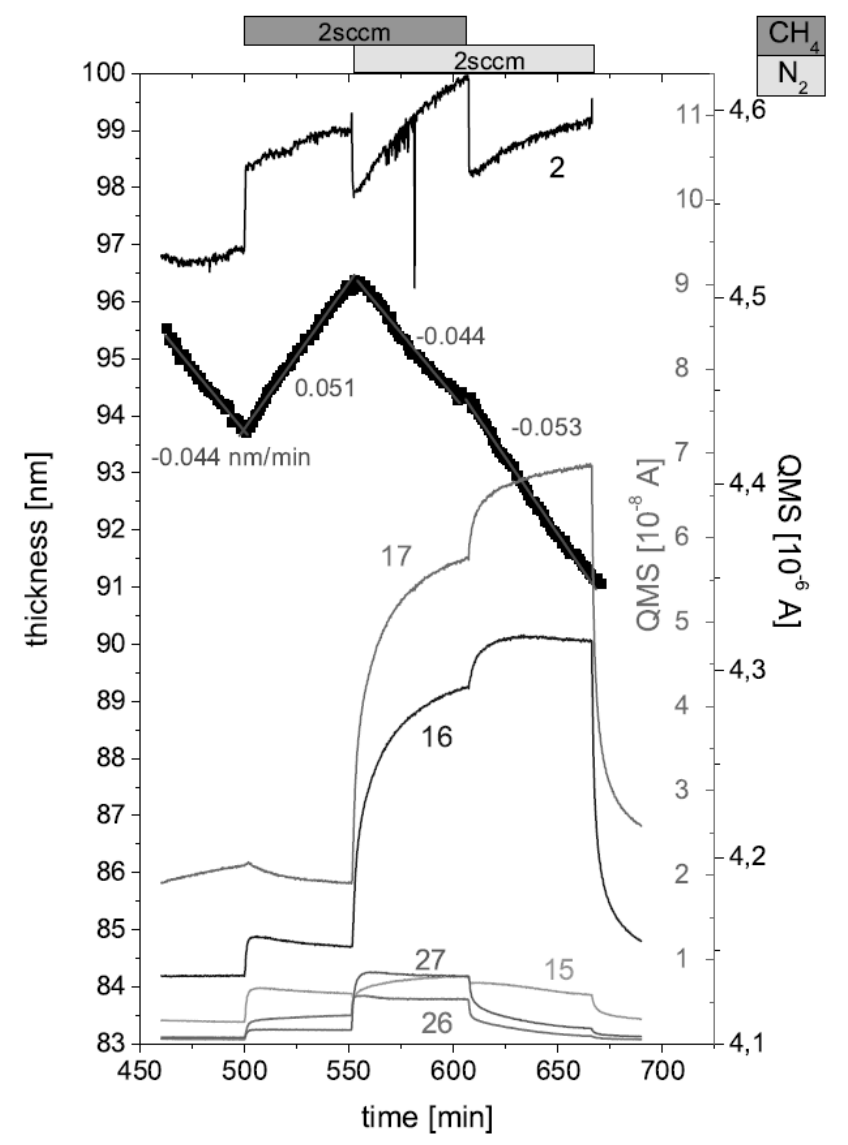

\begin{tabular}{cccccc}
\hline & 15 & 16 & 17 & 26 & 27 \\
\hline $\mathrm{NH}_{3}$ & $\mathrm{x}$ & $\mathrm{x}$ & $\mathrm{x}$ & - & - \\
$\mathrm{CH}_{4}$ & $\mathrm{x}$ & $\mathrm{x}$ & - & - & - \\
$\mathrm{C}_{2} \mathrm{H}_{\mathrm{x}}$ & $(\mathrm{x})$ & - & - & $\mathrm{x}$ & $\mathrm{x}$ \\
$\mathrm{HCN}$ & - & - & - & $\mathrm{x}$ & $\mathrm{x}$ \\
\hline
\end{tabular}

\section{REFERENCES}

[1] Counsell et al Plasma Phys. Control. Fusion 48 (2006) B189 - B199

[2] Waldmann, O. and Fussmann, G. 33rd EPS Conference on Plasma Phys. (2006) ECA Col. 301, P-1.049

[3] Jacob et al Appl. Phys. Lett. V.86 (2005) p.204103ff

[4] Kastelewicz, H. and Fussmann, G. Contrib. Plasma Phys. 44, No. 4, 352 - 360 (2004)

[5] Tabarés et al Plasma Phys. Control. Fusion 44 (2002) L37 - L42

[6] von Keudell et al Journal of Applied Physics 79 (2) p.1092 - 1098 (1996)

FIGURE 5. Left: QMS signal evolution and film thickness. Right: Cracking pattern excerpt for some relevant molecules. 